\title{
International Activities in Metrology in Chemistry
}

\author{
Robert I. Wielgosz* and Robert Kaarls
}

\begin{abstract}
In today's economy goods and information are being exchanged globally, international travel is commonplace as is the cross-boarder transport of livestock and agricultural products, and this trend is set to continue. Just as important are issues that impinge on our quality of life, such as health care, the environment and food quality. A strong international measurement and standards infrastructure is critical to assure equity in trade and a high quality of life, by ensuring that products and services meet their specifications. In the field of chemical measurements, certified reference materials (CRMs), measurement standards and reference measurement results provide stated references upon which analytical laboratories can anchor their measurement results. The traceability of measurement results to internationally accepted stated references, together with their stated measurement uncertainties, as described in ISO/IEC 17025, provides the basis for their comparability and global acceptance. Recent global activities which are succeeding in developing a system for the international acceptance of chemical measurements are described, notably: activities of the National Metrology Institutes and the BIPM; the Mutual Recognition Arrangement of the International Committee of Weights and Measures (CIPM MRA) for National Calibration Certificates and Measurement Capabilities; the Inter-Laboratory Comparisons organised through the working groups of the Consultative Committee for Amount of Substance - Metrology in Chemistry (CCQM), and the activities of the Joint Committee for Traceability in Laboratory Medicine (JCTLM).
\end{abstract}

Keywords: BIPM $\cdot$ Calibration $\cdot$ Metrology $\cdot$ Traceability $\cdot$ Uncertainty

\section{Introduction}

International trade, globalisation, quality of life, and cross-border issues are concerns of today. In today's economy goods and information are being exchanged globally. Just as important are issues that impinge on our quality of life, such as health care, the environment and food quality. A strong international measurement and standards infrastructure is critical to ensure equity in trade and a high quality of life, and to facilitate global recognition of measurements to promote international trade and the economic growth of a nation.

\footnotetext{
${ }^{\star}$ Correspondence: Dr. R. I. Wielgosz
}

Bureau International des Poids et Mesures BIPM

Pavillon de Breteuil

F-92312 Sèvres Cedex, France

Tel.: + 33145076251

Fax: + 33145342021

E-mail: rwielgosz@bipm.org
In such a climate, measurement disagreements between countries are viewed as an unacceptable barrier to trade. To overcome such problems it is necessary to have an international infrastructure within which it is possible to make comparable measurements. This is true for all areas of measurements including chemical ones. Such a system requires measurement standards that have long-term stability and are internationally recognised. The International System of Units (SI) represents such a system, and by the use of traceable measurements provides an international infrastructure for comparable measurements. An international programme for metrology in chemistry is extending this infrastructure to the field of chemical measurements.

\section{Metrology: The Science of Measurement}

Metrology is the science of measurement, embracing both experimental and theoretical determinations at any level of uncertainty in any field of science and technology. Within a robust metrological system the values of measurement standards and measurement results are linked via comparisons or calibrations which take into account the measurement uncertainty of the linking processes. Measurement uncertainty is the parameter associated with the results of a measurement, that characterises the dispersion of the values that could reasonably be attributed to the measurand. The ISO Guide to the Expression of Uncertainty in Measurement (GUM) and the EURACHEM guide on measurement uncertainty provide guidance on the evaluation of measurement uncertainty. ${ }^{[1,2]}$ The property of the result of a measurement or the value of a measurement standard whereby it can be related to stated references, usually national or international measurement standards, through an unbroken chain of comparisons all having stated uncertainties, is termed (metrological) traceability. Where these stated references are realisations of the SI units the term SI-traceable is used.

Traceability is the basis of the comparability of a measurement: whether the result of a measurement can be compared to the previous one, a measurement result a year ago, or to the result of a measurement performed anywhere else in the world.

Traceability is most often obtained by calibration, establishing the relation between the indication of a measuring instrument and the value of a measurement standard. In the field of analytical chemistry the term Certified Reference Material (CRM) is more often used than measurement standard. A CRM is a reference material, accompanied by a certificate, one or more of whose property values are certified by a procedure which establishes traceability to an accurate realisation of the unit in which the property values are expressed, and for which each certified value is accompanied 
by an uncertainty at a stated level of confidence. Metrological traceability may also be established to a reference method, defining the measurand and fixing a number of influence parameters, the results of which are expressed in SI units, and an approach that has been documented for the field of laboratory medicine. ${ }^{[3]}$

The Reference Materials Committee (REMCO) of the International Organization for Standardization (ISO) formed in 1975, has been active in developing a series of guides to support best practice in the production and certification of reference materials. The committee has developed two guides to assist in the establishment of facilities to produce and certify reference materials and ensure their quality. ISO Guide 34 outlines the requirements to be met by a CRM producer to demonstrate competence, and ISO Guide 35 provides guidance on general and statistical principles related to the certification of reference materials, and cites models for the homogeneity testing, stability testing, and the characterisation of a candidate CRM.

In the field of metrology in chemistry the role of a National Metrology Institute (NMI) involves establishing programmes which facilitate traceable measurements to be achieved, including the provision of certified reference materials, both as pure materials and calibration solutions as well as matrix reference materials for method validation or calibration in particular when the commutability of the reference material is an issue. Additionally NMIs may provide Reference Measurement Services, where customers require a traceable measurement value with small uncertainty, e.g. for the reference value assignment of a proficiency testing sample, or a manufacturer's primary calibrator.

\section{An International Infrastructure for Chemical Measurements}

The foundations of the International System of Units were set with the establishment of the Bureau International des Poids et Mesures (BIPM), an international Treaty organisation, at the signing of the Meter Convention in 1875.[4] The task of the BIPM is to ensure world-wide uniformity of measurements and their traceability to the SI, and to facilitate international collaboration and agreement on metrological issues. Today, there are 53 Member States of the BIPM and 27 Associate States and Economies of the General Conference on Weights and Measures (CGPM). Experts, drawn generally from national metrology institutes (NMIs), meet at the BIPM's Consultative Committees to reach international agreement on specific areas of metrology. It is noted that in many countries additional expert institutes are designated to carry out all or part of the tasks and responsibilities in the field of metrology in chemistry. These, so called Designated Institutes, act as the NMI within the defined and agreed field of metrology. All rights and obligations that apply to the NMIs are also applicable to the Designated Institutes (DIs). In this paper the term NMIs also includes the DIs.

The BIPM's Consultative Committee for Amount of Substance - Metrology in Chemistry (CCQM) is responsible for issues related to metrology in chemistry. ${ }^{[5]}$ Created in 1993 by the International Committee of Weights and Measures (CIPM), the CCQM has seven established working groups on organic analysis, inorganic analysis, gas analysis, electrochemical analysis bio-analysis, surface analysis and Key Comparisons and CMCs. An important part of the work of the CCQM has focused on investigating the required approach to establish a measurement infrastructure for chemistry based upon SI-traceable measurements. These discussions have led to the definition of primary methods and procedures of measurement, where a primary reference measurement procedure (also termed primary method of measurement) is a method/procedure having the highest metrological qualities, whose operation can be completely described and understood, and for which a complete uncertainty statement can be written down in terms of SI units. Later, a differentiation was made between primary direct methods, which measure the value of an unknown without reference to a standard of the same quantity, and primary ratio methods, which measure the value of a ratio of an unknown to a standard of the same quantity; its operation must be completely described by a measurement equation. In addition to having studied how these concepts may be applied in their specific areas, the CCQM working groups are involved in the identification, development and execution of a series of international comparisons that are the technical basis for the mutual recognition of measurement capabilities among the NMIs.

\section{Mutual Recognition of National Measurement Capabilities}

The CIPM Mutual Recognition Arrangement (CIPM MRA) established in October 1999 has now been signed by the representatives of 74 institutes - from 47 Member States, 25 Associates of the CGPM, and 2 international organisations - and covers a further 123 institutes designated by the signatory bodies. The text of the arrangement and the list of signatories are both available on the BIPM website. ${ }^{[6]}$
The CIPM MRA provides the framework for 'mutual recognition of national measurement standards, and of calibration and measurement certificates issued by national metrology institutes'. The objectives of the CIPM MRA are to: establish the degree of equivalence of measurement standards maintained by NMIs; to provide for the mutual recognition of calibration and measurement certificates issued by NMIs; thereby to provide governments and other parties with a secure technical foundation for wider agreements related to international trade, commerce and regulatory affairs. It is founded on the efforts of each individual national metrology institute to base its measurements and measurement uncertainties on SI units.

The CIPM MRA is underpinned by international comparisons that are known as key and supplementary comparisons, and will result in the publication of lists of calibration and measurement capabilities of the NMIs. The CIPM MRA is overseen by the Joint Committee of the Regional Metrology Organizations and the BIPM (JCRB). This committee includes representatives of the following regional metrology organisations (RMOs): APMP (for the Asia/ Pacific region), EURAMET (for Europe), SIM (for the Americas), COOMET (for the Euro-Asian region), and AFRIMETS (for Africa)

\section{The BIPM Key Comparison Database (KCDB)}

The principal output of the CIPM MRA is the BIPM key comparison database (KCDB), which can be found at the BIPM website (www.bipm.org). The database, which is maintained by the BIPM, contains Appendices A (signatories of the CIPM MRA), B (the results of international comparisons), $\mathrm{C}$ (Calibration and Measurement Capabilities) and D (a list of all international comparisons) of the CIPM MRA. The statements of the calibration and measurement capabilities (Appendix C) include a full description of the service in question, and, most importantly, the uncertainty with which it is offered to customers. These uncertainty statements are examined carefully during the process laid down in the CIPM MRA and are reviewed in the light of the results of key comparisons and other factors that contribute to the reliability of the measurements.

The statements of measurement capability of each NMI, supported by their performance in the key comparisons provide the framework for underpinning the international comparability of measurements. The traceability of results of measurements to these stated references provides the basis for their comparability. 


\section{Key Comparisons for Chemistry and BIPM Laboratory Activities}

The working groups of the CCQM have been active in organising key comparisons in the field of chemical measurements. Over the period 1997-2009, ninety-eight key comparisons have been nominated in addition to one hundred and fifty-seven pilot studies, equating to approximately twenty international comparisons run each year in the field of chemistry and the biosciences. The key comparisons cover the range of interest of the working groups, and have included: measurements of cholesterol, glucose, creatinine, calcium in serum; natural gas and automotive emission gas standards; gas standards for the monitoring of air pollutants and greenhouse gases; sulfur in fuels; drugs of abuse in urine; elemental and anion calibration solutions; organic calibration solutions including PAHs and PCBs; pure organic materials; heavy metals in rice, fish, oil, water, wine, sediment, tissues and algae; constituents of steel, nickel, copper and aluminium; organic contaminants in fish and sediments; DNA and protein quantification; trace elements in food and food supplements; $\mathrm{pH}$ primary buffers and electrolytic conductivity standards; thin film composition and thickness.

The data provided in a comparison report include the measurement results and their uncertainties, and a calculation of the degrees of equivalence between the measurements of the various laboratories and the reference value for the comparison.

Although the key comparison programme for chemistry is extensive, it will not be possible to cover all analytes and all matrices in a one to one fashion. Therefore in a number of CCQM working groups, strategies are being developed to undertake a limited number of comparisons which will evaluate the core competencies NMIs need to have in place to deliver their measurement services.

An example of a key comparison and the measurement capabilities that it can underpin is CCQM-K49, the determination of toxic and essential elements in bovine liver, which was conducted under the auspices of the CCQM Inorganic Analysis Working Group (IAWG). ${ }^{[7]}$ Measurands for this study consisted of elements that were expected to be relatively easy to determine ( $\mathrm{Fe}$ and $\mathrm{Zn}$ ), moderately difficult to determine ( $\mathrm{Se}, \mathrm{Cd}$ and $\mathrm{Pb}$ ), and very difficult to determine ( $\mathrm{Cr}$ and $\mathrm{As}$ ). All elements were present at naturally occurring levels, and in naturally occurring (non-spiked) forms in the material. Sixteen National Metrology Institutes (NMIs) submitted data for the study using a variety of analytical methods. The results of the comparison for zinc and arsenic are shown in Fig. 1a and Fig. 1b. The CCQM working group concluded that the results of the comparison could provide evidence for underpinning claims on measurement capabilities for the elements analysed in a number of matrix categories (Table), notably: Category 10 (Biological fluids and materials) with the possible exception of 10.5 (Bone). The bovine liver matrix also was a direct fit for Category 11 (Food) with the exception of 11.3 (GMOs). In addition, it was decided that if an NMI had aggressive digestion capabilities and experience, the comparison would underpin claims on measurements capabilities for the relevant elements in some matrices in Category 12 (Fuels), with the possible exception of 12.2 (Petroleum Products), Category 13 (Sediments, Soils, Ores and Particulates), with the possible exception of 13.3 (Ores), and Category 14 (Other), with the exception of
14.5 (Thin Films), 14.6 (Coatings), 14.9 (Adhesives) and the possible exception of 14.8 (Rubber).

The BIPM laboratories have established a programme of international comparisons for metrology in chemistry, in support of the strategic plans developed by the CCQM Working Groups, based on facilities that would not readily be supported at the National Level. Activities in the BIPM Laboratories are targeted in two areas: the International equivalence of

Table. National calibration and measurement capabilities for categories covered by metrology in chemistry

\begin{tabular}{|c|c|}
\hline Measurement Category & $\begin{array}{l}N^{\circ} \text { of Claims } \\
\text { in the } \mathrm{KCDB}\end{array}$ \\
\hline 1. Pure chemicals & 296 \\
\hline 2. Inorganic solutions & 330 \\
\hline 3. Organic solutions & 351 \\
\hline 4. Gases & 1641 \\
\hline 5. Water & 132 \\
\hline 6. $\mathrm{pH}$ & 96 \\
\hline 7. Electrolytic conductivity & 31 \\
\hline 8. Metals and alloys & 276 \\
\hline 9. Advanced materials & 56 \\
\hline $\begin{array}{l}\text { 10. Biological fluids and } \\
\text { materials }\end{array}$ & 332 \\
\hline 11. Food & 270 \\
\hline 12. Fuels & 47 \\
\hline 13. Sediments, soils & 422 \\
\hline 14. Other & 34 \\
\hline
\end{tabular}
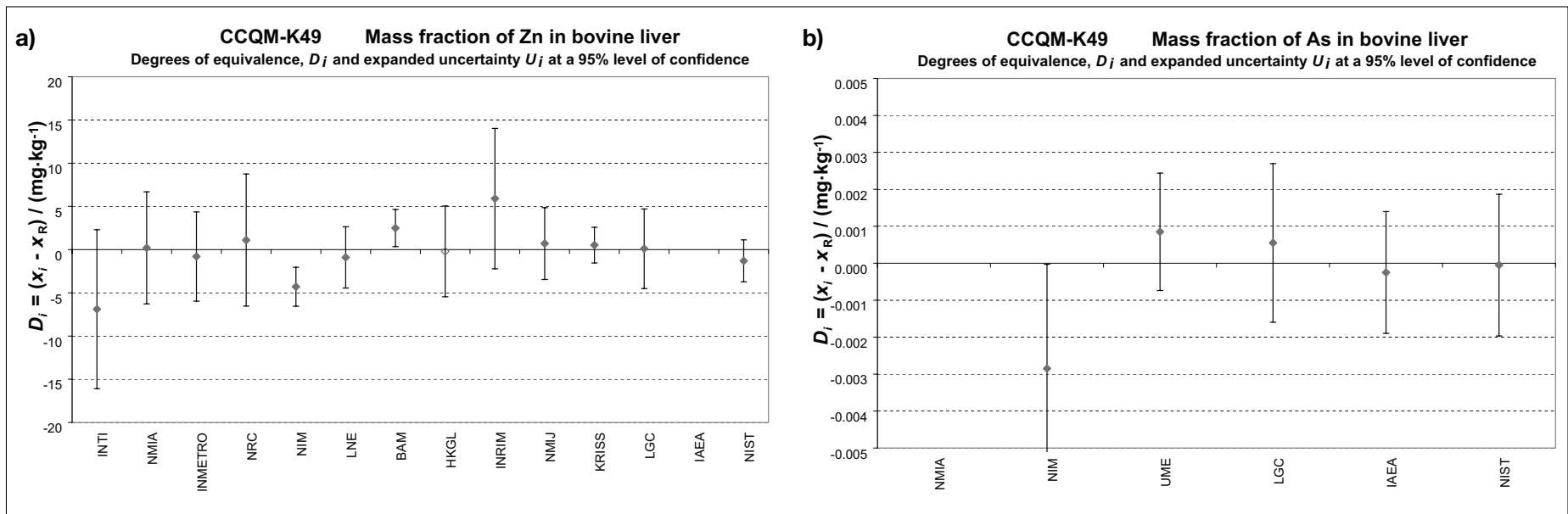

Fig. 1. a) CCQM-K49 results for zinc. The graph of equivalence is shown, with the difference between the laboratories' reported values and the reference value plotted together with the associated uncertainty of this difference. The nominal mass fraction of zinc in bovine liver was 180 $\mathrm{mg} \cdot \mathrm{kg}^{-1}$. b) CCQM-K49 results for arsenic. The graph of equivalence is shown, with the difference between the laboratories' reported values and the reference value plotted together with the associated uncertainty of this difference. The nominal mass fraction of arsenic in bovine liver was $0.02 \mathrm{mg} \cdot \mathrm{kg}^{-1}$. 


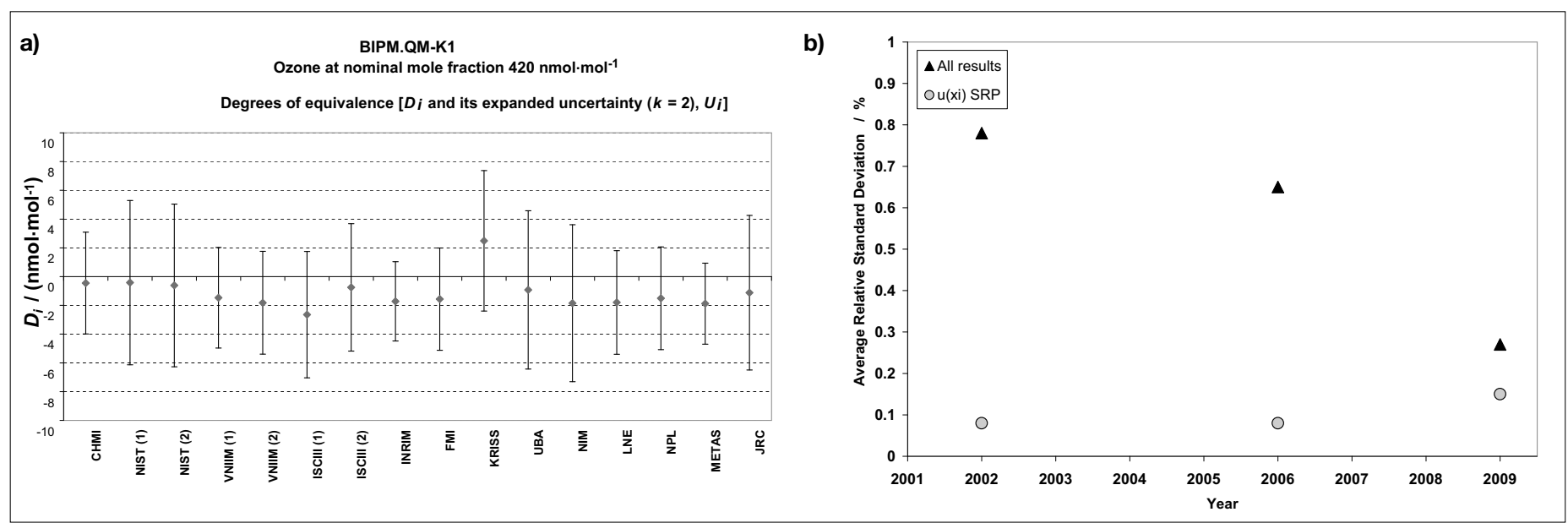

Fig. 2. a) Ozone Comparison Results. The graph of equivalence is shown, with the difference between the laboratories reported value and the reference value plotted together with the associated uncertainty of this difference. The nominal mass fraction ozone in air was $420 \mathrm{nmol}^{\prime} \mathrm{mol}^{-1}$, and all measurements were performed between January 2007 and December 2008. b) Improvement in laboratory performance for Surface Ozone Standards. The results of three comparisons are represented in the graph: EUROMET 414 performed in 2002; CCQM-P28 completed in 2006; BIPM. QM-K1 (first cycle) completed at the end of 2008. For all comparisons the relative spread of all participants' results, plotted as the relative standard deviation of all participants' results is compared to the standards uncertainty of a Standard Reference Photometer (SRP). Only the results of the BIPM.QM-K1 demonstrate a consistency between the stated uncertainty of the standard and the results of the comparison.

gas standards for air quality and climate change monitoring; and the International equivalence of primary organic calibrators, for health, food, forensics, pharma, and environmental monitoring. In the period 2005-2008 BIPM coordinated the following comparisons: CCQM-P28 (Surface ozone standards); CCQM-P73 (Nitrogen oxide standards - for emissions and air quality); BIPM.QM-K1 (Surface ozone); CCQM-P20.e (Theophylline - pure material calibrator); CCQM-P20.f (Digoxin - pure material calibrator); CCQM-K55.a (Estradiol - pure material calibrator). ${ }^{[8-10]}$ The results of BIPM.QM-K1 (2008/2009) are shown in Fig. 2a, where national ozone reference standards, used for underpinning air quality monitoring networks, are compared with the standards maintained at the BIPM. The level of agreement for the standards is now very good, and of sufficient consistency to enable reliable longterm monitoring of tropospheric ozone levels, having improved from previous comparisons, shown in Fig. 2b, following development work at the BIPM and the NIST to overcome systematic biases discovered in earlier comparisons. ${ }^{[11]}$

Thirty participations from NMIs in BIPM coordinated comparisons are expected each year. BIPM technical activities benefit the National Metrology Programmes in a number of ways:

i) They provide international comparisons and data to underpin all NMI Calibration and Measurement Capability Claims for Organic Primary Calibrators, currently $12 \%$ of all Chemical $\mathrm{CMCs}$, and by consequence underpin the traceability of measurement results in Clinical, Environmental, Food, Fo- rensic and Pharma application areas.

ii) They provide international facilities for the on-going comparison of surface ozone standards, as well as Nitrogen Monoxide, Nitrogen Dioxide, and Formaldehyde and thus support $\mathrm{Na}$ tional Air Quality Monitoring networks and Pollution Control Strategies.

iii) They will provide an international facility for the comparison of ambient methane gas standards, assuring the stability and reliability of measurements for the long term monitoring of the greenhouse gas, and their use in radiative and climate change models.

iv) They promote the development of higher order reference materials, methods/procedures and services by NMIs and their use by the IVD industry by maintaining the JCTLM Database of Reference Measurement Systems for Laboratory Medicine and the review process for nominated entries.

v) They are delivering a study on the measurement service and comparison needs for metrology in the biosciences and biotechnology, and thereby providing input into the formulation of $\mathrm{Na}$ tional Programmes in these fields of activity.

The BIPM laboratory programme responds to, and is in support of, the CCQM strategy for developing and maintaining a series of comparisons to underpin $\mathrm{Na}$ tional Measurement Capabilities. Important drivers to which the BIPM laboratory programme is responding and which will require the development and maintenance of an international infrastructure for metrology in chemistry and the biosciences include:
- climate change and air quality monitoring requirements and the control of emissions that lead to global warming and air pollution, which are being incorporated into national legislation and have been identified by the major intergovernmental or international bodies concerned such as the WMO, the Intergovernmental Panel on Climate Change, and UN Agencies;

- the development of a measurement infrastructure to support the implementation of a hydrogen economy and the use of biofuels;

- requests for measurement programmes to support traceability requirements from the clinical, food, forensic, sports drug testing and pharmaceutical communities;

- advances in measurement technologies allowing the full characterisation of biologicals by physico-chemical means, and the subsequent requirement for reference measurement systems for the diagnostic and therapeutic communities to improve the accuracy, comparability and reliability of measurement results used in diagnosis and healthcare.

The future strategy developed by the CCQM's Gas Analysis working group (GAWG), envisions the regular execution of core and analytical challenge comparisons to underpin National Measurement Capabilities. Analytical Challenge comparisons include species for which the laboratory measurement performance cannot be inferred between species. Comparisons within this category require dedicated facilities. Species for which there is an international requirement to maintain such facilities include: 
- greenhouse gases, including methane, with renewed commitments at the governmental level to address climate change, often through regulation for emissions with their impact assessed through long-term ambient monitoring;

- air quality relevant compounds such as formaldehyde or other volatile aromatic hydrocarbons which have been identified in National Air Quality Strategies and the WMO-GAW Strategic Plan;

- trace species in hydrogen fuel, which have been identified as critical parameters to control for the success of the hydrogen economy; impurities such as ammonia and organic sulphur compounds will poison fuel cells, reducing their lifetime or rendering them inoperable.

The CCQM GAWG has organised comparisons for the range of gases relevant to air quality, notably $\mathrm{NO}, \mathrm{NO}_{2}, \mathrm{O}_{3}$, $\mathrm{CO}, \mathrm{SO}_{2}$ and $\mathrm{C}_{6} \mathrm{H}_{6}$. Fig. 3 summarises the comparisons carried out under the auspices of the CCQM GAWG for nitrogen monoxide, from mole fractions which are relevant to the monitoring of emissions, down to concentrations that are relevant to the monitoring of air quality and pollution and concentrations found in urban areas. The Calibration and Measurement Capability Claims of an NMI providing standards across this range of concentrations is shown, and compared with the uncertainty of the degree of equivalence with the reference value for each comparison, indicating that the performance claimed by the laboratory is consistent with its performance in international comparisons.
The CCQM Organic Analysis Working Group's primary focus is the critical evaluation and benchmarking of NMI capabilities for the execution of 'higher order' measurement procedures for well-defined organic molecular entities for which the SI-traceable amount of substance is to be determined. In carrying out these activities over the last ten years the working group has undertaken twenty key comparisons targeting 50 measurands and 41 pilot studies on 126 analyte-matrix combinations. The OAWG is developing a strategy to limit the number of international comparisons required to underpin a broad range of measurement capabilities, by focusing comparisons on core competencies rather than individual species. The value assignment of pure substance primary calibrators is the basis of traceability within the organic field, and is one of the core competencies identified by the OAWG, and which is being underpinned by comparisons coordinated by the BIPM Laboratories. A recent survey of NMI programmes identified that national measurement services would be developed to underpin traceability requirements in the food and clinical areas in addition to activities in environmental monitoring, forensics and pharma. The BIPM coordinated comparisons will continue to underpin all Organic Calibrator claims in the organic field, based on a model that tests principal methods of purity analysis depending upon the molecular weight and polarity of analytes. Analytes for the comparisons are selected because of their importance to NMI programmes and measurement systems for particular sectors in addition to their physico-chemical

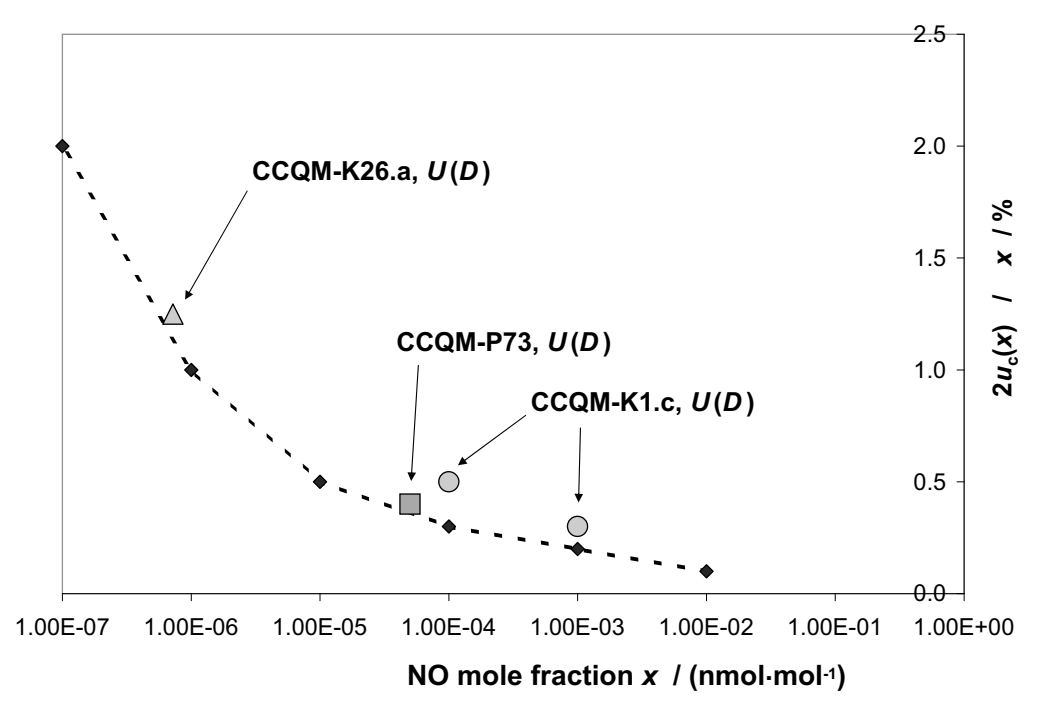

Fig. 3. NO comparison results and CMC claims. The expanded uncertainty within CMC claims of an NMI for standards of nitrogen monoxide in nitrogen over the mole fraction range $0.01 \mathrm{~mol}$. $\mathrm{mol}^{-1}$ to $100 \mathrm{nmol} \cdot \mathrm{mol}^{-1}$ are plotted (dashed line) and compared to the uncertainty in the degree of equivalence $U(D)$ achieved by that laboratory in three comparisons (CCQM-K1.C, CCQM-P73 and CCQM-K26.a). The uncertainties claimed by the laboratory are consistent with their performance in the comparisons. properties. Primary calibrators of particular importance for the future comparisons include:

- monitored therapeutic drugs and vitamins, required for the establishment of Reference Measurement Systems in Laboratory Medicine, and to enable the in vitro diagnostic devices industry to meet measurement traceability requirements within regulations;

- contaminants and residues within foods including food contact materials, required to meet traceability and uncertainty requirements for maximum permissible limits;

- active ingredients and impurities within pharmaceutical products and herbs and botanicals, for compliance with the safety and efficacy requirements of regulations.

One of the most recently formed working groups of the CCQM has been on surface analysis. Its first comparison, CCQM-K32/P84 (Gate oxide, $\mathrm{SiO}_{2}$ on $\mathrm{Si}$ ), addressed the need for traceable determinations of the thickness of gate oxides in the semiconductor industry. A suite of nine samples of nominal thickness in the range $1.5 \mathrm{~nm}$ to $8 \mathrm{~nm}$ on (100) and (111) Si substrates have been analysed in the comparison. Eleven NMIs produced results that were in good agreement at $1.5 \mathrm{~nm}$ surface thickness with an expanded uncertainty of $1.5 \%$ relative. As a consequence of this study, the first CMCs in surface analysis have been claimed by a number of NMIs. Other comparisons currently being coordinated by the group included: CCQMK67/P108 (Amount of $\mathrm{Fe}$ and $\mathrm{Ni}$ in $(200$ $\mathrm{nm}$ ) Fe-Ni alloy film on Si); CCQM-P80 (Carbon in precipitates in Fe); CCQM-P81 (Nitrogen in surface layers of Fe); CCQMP95 (Determination of nitrogen in doped diamond like carbon (DLC) films).

The Electrochemical Analysis Working Group (EAWG) comparisons have concentrated on $\mathrm{pH}$ and electrolytic conductivity, and for $\mathrm{pH}$ now span the range 1.5 to 10 . Similarly, the suite of key and pilot studies undertaken for electrolyte conductivity span nearly five orders of magnitude with seawater on the one extreme and pure water on the other although lying 100-fold below the lowest conductivity comparisons undertaken to date.

The CCQM Bio-analysis Working Group (CCQM-BAWG) is assessing measurement capability and comparison needs in a number of areas, notably: nucleic acids; proteins; cells and tissues; epigenomics; nano-biotechnology and polysaccharides. The first CMC claim covered by the comparisons coordinated by the Working Group has been made based on CCQMK61 (Quantitative PCR Calibration), and underpins key DNA measurements. International comparisons carried out by the 
BAWG include: CCQM-P113 (Relative Quantification of Genomic DNA Fragments); CCQM-P59.1 (Protein Structural Measurements by Circular Dichroism); CCQM-P101 (Protein Glycosylation) identifying and determining relative quantities of glycan species in a mixture typical of that released from therapeutic glycoproteins; CCQM-P58.1 (Comparability of Fluorescence in ELISA); CCQM-P94.1 (Quantification of DNA Methylation); CCQM-P102 (Quantification of Cells with Specific Phenotypic Characteristics;) and CCQM-P103 (Measurement of a Multiplexed Panel of RNA Transcripts).

\section{A Searchable Database of National Chemical Calibration and Measurement Capabilities}

A searchable database of the Calibration and Measurement Capabilities (CMCs) of NMIs for chemical measurements is included in Appendix $\mathrm{C}$ of the CIPM MRA, listed under Amount of Substance.

The chemical categories to be covered are: high-purity chemicals; inorganic solutions; organic solutions; gases; water; metals and metal alloys; advanced materials; biological fluids and materials; food; fuels; sediments; soils; ores and particulates; $\mathrm{pH}$ and electrolytic conductivity; and other materials. As of 8 April 2009, there were 4314 CMCs published in the chemical categories of the BIPM key comparison database (KCDB), and distributed across these categories as indicated in the Table.

In searching the database, the user is able to select a country name and a chemical category from lists of all the countries and chemical categories for which $\mathrm{CMCs}$ have been declared. Additionally a keyword search is available for entries related to a specific analyte. The search can be performed on the name (complete or partial) of the desired analyte. The results of a search for 'cholesterol', for example, will include the concentration range and uncertainty at which this service is delivered within the chosen country or countries. The traceability of measurement results to these stated references provides the basis for their comparability.

An important objective for the database is for it to be utilised by accredited laboratories for the acceptance of one another's results. One of the aims of a mutual recognition arrangement being established by the International Laboratory Accreditation Cooperation (ILAC) is for signatories to recognise one another's accredited laboratories. ${ }^{[12]}$ In this arrangement, one of the principle elements in establishing confidence among the participating systems is confidence in the metrology institutes of

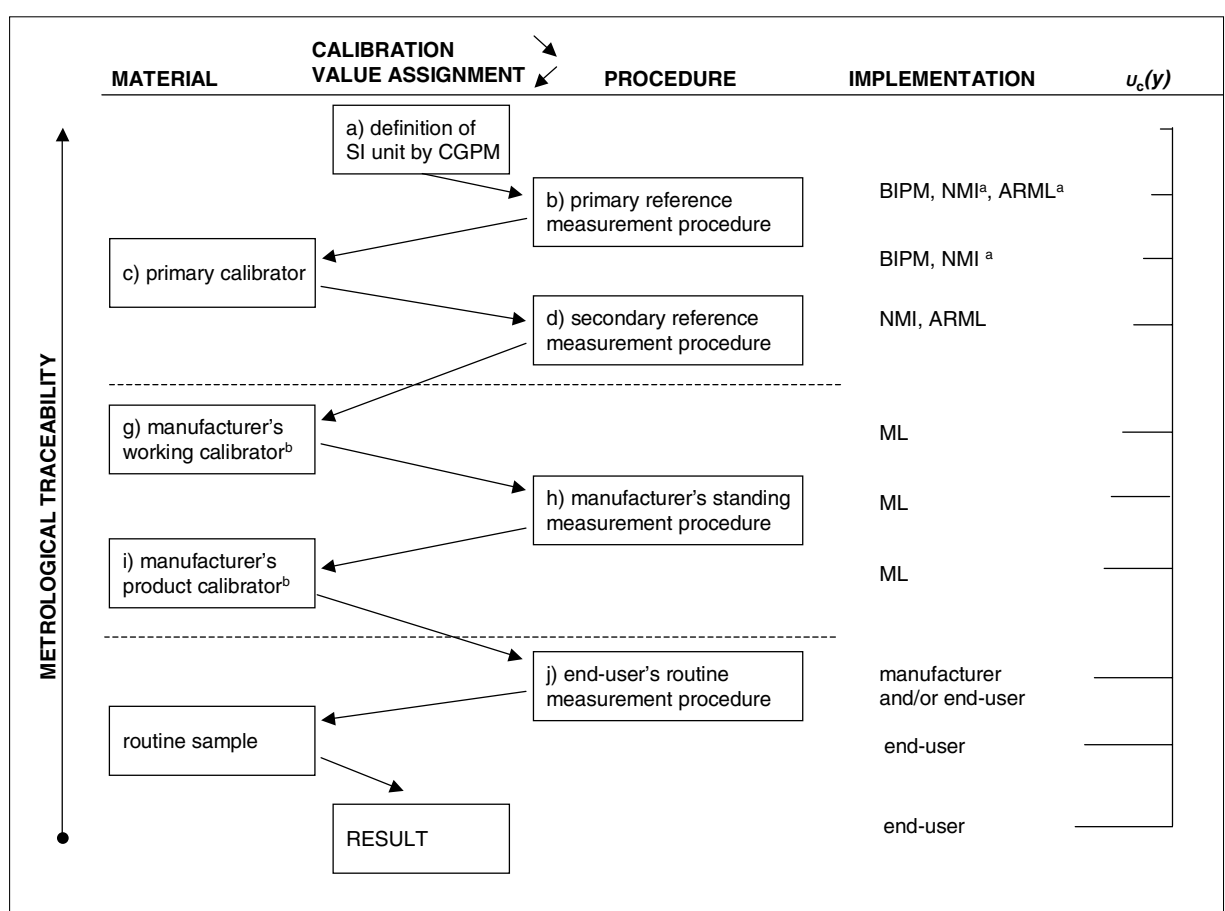

Fig. 4. Calibration hierarchy and metrological traceability to the SI, from ISO17511:2003 In vitro diagnostic medical device - Measurement of quantities in biological samples - Metrological traceability of values assigned to calibrators and control materials. (Reproduced with permission from the International Organization for Standardization, ISO. This standard can be obtained from any ISO member and from the website, www.iso.org; () ISO.)

the signatory economies to which traceability is claimed by accredited laboratories. The KCDB provides the technical basis for this to be realised.

\section{Reference Measurement Systems for Laboratory Medicine}

The need for reliable and reproducible measurement results in the diagnostic field is well understood, with, for example, the Scientific Division of the International Federation of Clinical Chemistry and Laboratory Medicine (IFCC), representing professionals in the field, developing reference methods for key measurands. The importance of metrological traceability for diagnostic measurements has also been recognised in legislation with the implementation of the European Directive 98/79/ $\mathrm{EC}$ on in vitro diagnostic medical devices, which included the requirement for the traceability of values assigned to calibrators and control materials for in vitro diagnostic devices to be assured through available reference measurement procedures and services and/or reference materials of higher order, which collectively constitute Reference Measurement Systems for laboratory medicine. The concept of metrological traceability is particularly well developed in the field of laboratory medicine, and ISO 17511 'In vitro diagnostic medical devices - Measurement of quantities in biological samples - Metrological traceability of values assigned to calibrators and control materials', describes reference measurement systems, which ensure traceability to the SI via reference measurement procedures and certified reference materials. A calibration hierarchy with traceability to the SI is depicted in Fig. 4.

The European Directive 98/79/EC on in vitro diagnostic medical devices was a major driving force for the establishment of the Joint Committee for Traceability in Laboratory Medicine (JCTLM), by the Bureau International des Poids et Mesures (BIPM), together with the International Federation of Clinical Chemistry and Laboratory Medicine (IFCC) and the International Laboratory Accreditation Cooperation (ILAC). The JCTLM has established a framework which lays down a process whereby reference materials and reference measurement procedures are examined with respect to conformity with appropriate international documentary standards (ISO 17511, 15193 and 15194).

The outputs of the framework are databases of available higher-order reference materials and higher-order reference measurement procedures as well as reference laboratories (operating in compliance with ISO 15195 and 17025) that can be used by the IVD industry and other users to meet requirements for traceability for in vitro diagnostic and laboratory medicine measurements. 
The database classifies entries for Higher Order Reference Materials and Reference Measurement Procedures in two lists (http://www.bipm.org/jctlm/):

List I: Certified Reference Materials and Reference Measurement Procedures for well-defined chemical entities or internationally recognised reference methoddefined measurands. Reference Materials and Measurement Procedures included in this category are those that provide values that are traceable to the SI units; e.g. electrolytes, enzymes, drugs, metabolites and substrates, non-peptide hormones, and some proteins.

List II: International Conventional Reference Materials, i.e. where the measurand(s) is/are not SI traceable and/ or no internationally recognised reference measurement procedure is available; e.g. WHO reference materials for coagulation factors, nucleic acids, and some proteins.

As of May 2009, the JCTLM Database contained:

- 208 available certified reference materials that cover nine categories of analytes. Amongst these reference materials 33 are currently listed as List II reference materials;

- 146 reference measurement methods or procedures that represent about 75 different analytes for eight categories of analytes;

- and, 128 reference measurement services that can be delivered by 16 reference laboratories from 10 countries covering 6 categories of analytes.

\section{Links with Other International Programmes and Stakeholders}

A number of international organisations and industrial sectors have stringent measurement requirements. The CCQM continues to make efforts to meet these requirements without duplicating activities already underway. CCQM activities have included:

i) the organisation of sector specific workshops to identify measurement, traceability and standards needs;

ii) and encouraging the participation of organisations in CCQM comparisons as working group members or as expert laboratories.

Recent workshops organised by the CCQM have included:

i) Reference Measurement Systems for Food Analysis (2004), with participation from the Codex Alimentarius Commission, International Organisation of Vine and Wine (OIV), International Olive Oil Council (IOOC), and the International Dairy Federation (IDF); ii) Higher-Order measurement methods for physiologically-significant molecules (2005), with participation from the National Institute of Biological Standards and Control (NIBSC) UK, a WHO Standards Custodian laboratory; iii) Pharma and Bio-Pharma Workshop (2008), with participation from the Unites States Pharmacopeia (USP) and the European Directorate for the Quality of Medicines and Healthcare (EDQM).

Furthermore, measurement and measurement standard requirements have been presented to the CCQM by the World Anti Doping Agency (WADA); the European Network of Forensic Science Institutes (ENFSI); the World Meteorological Organization's Global Atmosphere Watch (WMO-GAW) programme and the International Federation of Clinical Chemistry and Laboratory Medicine (IFCC) and the World Health Organization (WHO). Expert laboratories from other international organisations have also participated in CCQM comparisons, notably: WMOGAW World Calibration Centres and Central Calibration laboratories in CCQM-P41 (Greenhouse gases, carbon dioxide and methane at ambient levels), CCQM-P28 (Ozone at ambient levels), CCQM-K68 $\left(\mathrm{N}_{2} \mathrm{O}\right.$ at ambient levels); United States Pharmacopeia (USP) in CCQM-P20.e (Theophylline - pure material calibrator), CCQM-P20.f (Digoxin - pure material calibrator), CCQM-K55.a (Estradiol - pure material calibrator), CCQM-P110 (Glycan species measurement in digest of glycoproteins); NIBSC in CCQM-P110 (Glycan species measurement in digest of glycoproteins) and CCQM-P102 (Quantification of Cells with specific phenotype characteristics).

The comparisons CCQM-P68 (Anabolic steroids in urine: 19-norandrostone in human urine), CCQM-K69 (Anabolic steroids in urine: Testerone glucuronide), CCQM-P115 (Anabolic steroids in urine: Testerone glucuronide and epitestosterone glucuronide) were all performed on materials that were candidate CRMs for the WADA.

By continuing to maintain close cooperation with the experts in the different sectors CCQM can ensure that its activities remain fit-for-purpose and satisfy needs in trade, industry and society, adding to economic welfare and to the quality of life.

\section{Conclusions}

The work of the CCQM and the implementation of the CIPM MRA are providing an infrastructure which enables the comparability of national chemical standards and calibration and measurement services to be demonstrated and this has been strengthened in the area of laboratory medicine through the work of the JCTLM. The data contained with the BIPM key comparison and calibration database and the JCTLM database will facilitate the acceptance of calibration or measurement certificates, providing confidence in chemical measurements, and facilitating equity in trade and a high quality of life, by ensuring that products and services meet their specifications.

Received: June 25, 2009

[1] 'Evaluation of measurement data - Guide to the expression of uncertainty in measurement', JCGM 100: 2008.

[2] 'EURACHEM/CITAC Guide CG4 Quantifying Uncertainty in Analytical Measurement', Eds. S. L. R. Ellison, M. Rösslein, A. Williams, 2nd Ed., 2000.

[3] ISO17511:2003, 'In vitro diagnostic medical device - Measurement of quantities in biological samples- Metrological traceability of values assigned to calibrators and control materials'.

[4] www.bipm.org.

[5] R. Kaarls, T. J. Quinn, Metrologia 1997, 34, 1.

[6] http://www.bipm.org/en/cipm-mra/.

[7] R. R. Greenberg, Metrologia 2008, 45, Tech. Supp., 08016.

[8] J. Viallon, P. Moussay, M. Esler, R. Wielgosz, W. Bremser, J. Novák, M. Vokoun, A. Botha, M. J. Van Rensburg, C. Zellweger, S. Goldthorp, A. Borowiak, F. Lagler, J. Walden, E. Malgeri, M. P. Sassi, P. M. Gomez, R. F. Patier, D. G. Madruga, J.-C. Woo, Y. D. Kim, T. Macé, C. Sutour, A. Surget, B. Niederhauser, D. Schwaller, B. Frigy, I. G. Váraljai, S. Hashimoto, H. Mukai, H. Tanimoto, H. P. Ahleson, A. Egeløv, N. Ladegard, L. Marsteen, K. Tørnkvist, F. R. Guenther, J. E. Norris, T. L. Hafkenscheid, M. M. Van Rijn, P. Quincey, B. Sweeney, S. Langer, B. Magnusson, J. Bastian, V. Stummer, M. Fröhlich, A. Wolf, L.A. Konopelko, Y. A. Kustikov, D. V. Rumyanstev, Metrologia 2006, 43, Tech. Supp., 08010.

[9] R. I. Wielgosz, M. Esler, J. Viallon, P. Moussay, S. H. Oh, B. M. Kim, A. Botha, J. Tshilongo, I. S. Mokgoro, M. Maruyama, T. Mace, C. Sutour, V. Stovcík, M. Valková, S. Musil, A. Pérez Castorena, V. Serrano Caballero, F. Rangel Murillo, L. A. Konopelko, Y. A. Kustikov, V. V. Pankratov, E. V. Gromova, W. J. Thorn, F. R. Guenther, D. Smeulders, G. Baptista, F. Dias, R. M. Wessel, G. Nieuwenkamp, A. M. H. van der Veen, Metrologia 2008, 45, Tech. Supp., 08002.

[10] J. Viallon, P. Moussay, R. Wielgosz, B. Niederhauser, 'Final report, on-going key comparison BIPM.QM-K1: Ozone at ambient level, comparison with METAS, 2008', Metrologia, 2009, 46, Tech. Supp., 08016.

[11] J. Viallon, P. Moussay, J. E. Norris, F. R. Guenther, R. I. Wielgosz, 'A study of systematic biases and measurement uncertainties in ozone mole fraction measurements with the NIST Standard Reference Photometer', Metrologia 2006, 43, 441.

[12] http://www.ilac.org. 